\title{
ASSOCIATION BETWEEN CHOLESTEROL INTAKE AND ALL-CAUSE MORTALITY: NHANES-LINKED MORTALITY STUDY
}

\author{
Xiangpeng Du, ${ }^{1,2}$, Hui Xin ${ }^{1}$ \\ 1'Department of Cardiology, Hospital of Qingdao University, Qingdao, Shandong, China \\ ${ }^{2}$ Department of Cardiology, Weihaiwei People's Hospital, Weihai, Shandong, China
}

\begin{abstract}
SUMMARY
Objectives: There has been insufficient evidence for a quantitative recommendation for dietary cholesterol, therefore, we aim to investigate the optimal cholesterol intake related to a lower all-cause mortality risk.

Methods: The National Health and Nutrition Examination Survey (NHANES) is a large population survey to investigate public health in the United States. We analysed data from 1999-2002 linked with mortality data obtained through 2006. Cox proportional hazard ratios (HRs) with 95\% confidence intervals (Cls) were estimated to assess risks for all-cause mortality associated with cholesterol intake.

Results: A total of 7,728 participants were analysed in the present study, and 519 assumed death events. Compared with the third quartile (216-373 mg/day) of cholesterol intake, the risks of mortality increased in both the first two and the last quartiles (quartile 1: $\mathrm{HR} 1.53,95 \% \mathrm{Cl}$ 1.16-2.00; quartile 2: HR 1.22, 95\% $\mathrm{Cl} 0.94-1.60$; quartile 4: $\mathrm{HR} 1.39,95 \% \mathrm{Cl} 1.05-1.83$ ). The association between cholesterol intake and the risk of all-cause mortality followed a U-shaped curve, with the cholesterol intake associated with the lowest mortality being $328 \mathrm{mg} /$ day.

Conclusions: The present study suggests an optimal cholesterol intake for lowering the all-cause mortality risk.
\end{abstract}

Key words: cholesterol intake, all-cause mortality, NHANES, U-shaped curve, dietary

Address for correspondence: Hui Xin, Department of Cardiology, Affiliated Hospital of Qingdao University, 16\# Jiang-Su Road, Qingdao, Shandong, China. E-mail: dynasterzm|@163.com

https://doi.org/10.21101/cejph.a6276

\section{INTRODUCTION}

Given high serum cholesterol level is an independent risk factor of major events, such as cardiovascular disease (CVD), CVD mortality or all-cause mortality (1-3). Dietary cholesterol has been considered to be the less the better. However, the results of relevant studies showed controversial findings. Previous research mostly found that higher dietary cholesterol or fat intake was related to higher number of major events $(4,5)$, while recently the Prospective Urban Rural Epidemiology (PURE) study showed that higher intake of total fat and individual types of fat (including saturated fat, monounsaturated fat, and polyunsaturated fat) was related to lower total mortality risk, and not associated with CVD or CVD mortality (6).

Generally, as the main fat-dietary product, cholesterol was still without adequate evidence for a quantitative limit of dietary intake. The 2015 Dietary Guidelines Advisory Committee (DGAC) report (7) shifted the previous limit of a maximum of $300 \mathrm{mg} /$ day for dietary cholesterol intake, and meanwhile stated that people should eat as little dietary cholesterol as possible, suggesting that cholesterol is considered to be important in building a healthy eating style, and healthy cholesterol intake should be neither too little nor too much. In the present study, we link the National Health and Nutrition Examination Survey (NHANES) dietary data with mortality information to evaluate the optimal cholesterol intake related to lower all-cause mortality risk.

\section{MATERIALS AND METHODS}

\section{Data Source}

The NHANES is a population survey implemented by the Centers for Disease Control and Prevention (CDC) to investigate public health in the United States. Details about NHANES are available elsewhere $(8,9)$, including the protocol and data collection procedures. An already published NHANES data package (including the 1999-2000, 2001-2002, 2003-2004, and 2005-2006 datasets) $(10,11)$ was used in the present study, in which the mortality information was collected from the data of the survey participants and ascertained via probabilistic matching between the NHANES and the National Death Index (NDI) death certificate information.

We used the 1999-2000 and 2001-2002 datasets to analyse the association between cholesterol intake and all-cause mortality. A total of 21,004 participants $(9,965$ in the 1999-2000 and 11,039 in the 2001-2002 survey) were included in the two surveys, 9,563 participants aged under 18 and 3,713 participants with missing 
values were excluded, and 7,728 participants were analysed in the present study.

\section{Variables Description}

Variables in the present study include dietary (cholesterol, carbohydrate, total energy, and alcohol) intake, age, gender, race, body mass index (BMI), smoking history, chronic disease history, income, education, and physical activity. Covariate alcohol intake was divided into three groups: $0 \mathrm{gm} /$ day, $\leq 30 \mathrm{gm} /$ day, and $>30$ gm/day. Smoking status included both active and passive smoking. Covariate income, which was originally categorized into eleven groups ( $\$ 0$ to $\$ 4,999 ; \$ 5,000$ to $\$ 9,999 ; \$ 10,000$ to $\$ 14,999$; $\$ 15,000$ to $\$ 19,999$; $\$ 20,000$ to $\$ 24,999$; $\$ 25,000$ to $\$ 34,999$; $\$ 35,000$ to $\$ 44,999 ; \$ 45,000$ to $\$ 54,999 ; \$ 55,000$ to $\$ 64,999$; $\$ 65,000$ to $\$ 74,999 ; \$ 75,000$ and over), was combined into three new groups: $\$ 0$ to $\$ 24,999 ; \$ 25,000$ to $\$ 54,999 ; \$ 55,000$ and over. Covariate chronic disease history includes diabetes, coronary disease, hypertension, asthma, rheumatoid arthritis, osteoarthritis, and 30 site-specific cancers (such as breast, skin, lung, colon, bladder, kidney, and other type of cancers). Physical activity was treated as an ordinal factor based on physical activity guideline categories for no aerobic activity, low activity (medium intensity activity more than baseline but fewer than $150 \mathrm{~min} /$ week), moderate activity (150 to 300 medium intensity $\mathrm{min} / \mathrm{week}$ ) and high activity ( $>300 \mathrm{~min}$ medium intensive activity per week or $>150$ min high intensity per week). Other variables were kept from the original data.

\section{Statistical Analysis}

Continuous variables were expressed as the mean $\pm \mathrm{SD}$ or median (interquartile range, IQR). Categorical variables were expressed as percentages and numbers. Generalized linear models were applied to evaluate the trends of baseline characteristics across quartiles of cholesterol intake (quartile $1<124 \mathrm{mg}, 124 \leq$ quartile $2<216 \mathrm{mg}, 216 \leq$ quartile $3<373 \mathrm{mg}$, quartile $4 \geq 373$ $\mathrm{mg}$ ). Cox proportional hazard models were performed to obtain the hazard ratios and their $95 \%$ confidence intervals (CIs) in univariate model and after adjustment for total energy, carbohydrate and alcohol intake, age, gender, race, BMI, chronic disease history, smoking status, income, education, and physical activity. Moreover, we fitted the multivariate model with quadratic term of cholesterol intakes as well as restricted cubic splines (RCSs) for non-linear relationship between cholesterol intake and all-cause mortality and calculated the optimal cholesterol intake. Statistical relationships were considered significant for $\mathrm{p}$-values $<0.05$. All analyses were performed using R software, version 3.4.4.

\section{RESULTS}

The present study includes 7,728 subjects (age: $45.7 \pm 19.6$ years, male/female 3,623/4,105). Of those, 519 were assumed death events. The median follow-up period was 68 months. Characteristics of the study population are listed in Table 1. As cholesterol intake increased, the intake of total energy, carbohydrate and alcohol all significantly increased ( $\mathrm{p}$-value for trend < $0.001)$, while age and chronic disease prevalence significantly decreased ( $\mathrm{p}$-value for trend $<0.001$ ).
In both crude and multivariate models (adjustment for intake of total energy, carbohydrate, and alcohol, age, gender, race, BMI, chronic disease history, smoking status, income, education, and physical activity), the third quartile of cholesterol intake appeared to have the lowest risk of all-cause mortality. Compared with the third quartile $(216 \leq$ cholesterol intake $<373 \mathrm{mg}$ /day) of cholesterol intake, the risk of mortality increased by $53 \%$ in the first quartile (cholesterol intake $<124 \mathrm{mg} /$ day), tended to increase in the second quartile $(124 \leq$ cholesterol intake $<216 \mathrm{mg} /$ day $)$, and increased by $39 \%$ in the fourth quartile (cholesterol intake $\geq 373 \mathrm{mg} /$ day). Compared with the first quartile, the risk of mortality decreased by $20 \%$ in the second quartile, decreased by $35 \%$ in the third quartile, and tended to decrease in the fourth quartile (Table 2).

The association between cholesterol intake and the risk of allcause mortality followed a U-shaped curve, with increased risk above and below the reference range of 216 to $373 \mathrm{mg}$ (Table 2). In Figure 1, after excluding the top and bottom 5 percentiles of cholesterol intake, the RCS also demonstrated a U-shaped association between cholesterol intake and the risk of all-cause mortality. We also added a quadratic term of cholesterol intake in the model, using the quadratic and linear coefficients to calculate the optimal cholesterol intake, and found the cholesterol intake associated with the lowest mortality was $328 \mathrm{mg} /$ day in the multivariate model.

\section{DISCUSSION}

This study contributes some valuable findings. First, it showed that the association between cholesterol intake and the risk of all-cause mortality followed a U-shaped curve, and the optimal cholesterol intake appeared to be around $300 \mathrm{mg} /$ day. Second, compared with the first quartile (cholesterol intake $<124 \mathrm{mg}$ / day), the other quartiles showed lower mortality risks, suggesting that the least intake of dietary cholesterol was associated with the highest risk. We concluded that healthy dietary cholesterol intake is around $300 \mathrm{mg} / \mathrm{day}$ and excessive restrictions of cholesterol intake are harmful to the health.

Previous studies proved that high serum cholesterol level was associated with high risks of major events (CVD, CVD mortality or all-cause mortality) $(2,3)$. Therefore, in the past several years, cholesterol has been thought to be a nutrient of concern for overconsumption. Previously, the DGAC recommended that cholesterol intake should be limited to $300 \mathrm{mg}$ /day (equivalent to $1.5 \mathrm{eggs} /$ day), and lower in red and processed meat $(7,12)$. However, recent studies have come to opposite conclusion - there is no appreciable relationship between consumption of dietary cholesterol and serum cholesterol (13), and high intake of cholesterol or cholesterol-rich foods is not related to high risk or even lowers the risk of major events $(6,14,15)$. It appears that dietary cholesterol is a highly controversial nutrient. As well, the 2015-2020 DGAC report did not bring forward the recommendation of cholesterol intake limit, waiting for further studies to generate more consistent evidence of quantitative recommendation of cholesterol intake.

In the present study, we demonstrated the U-shaped association between dietary cholesterol and all-cause mortality, and we believed that healthy cholesterol intake should be neither too little nor too much. However, there are still some limitations: we analysed data from NHANES 1999-2004, which did not include 
Table 1. Baseline characteristics of participants, NHANES 1999-2002 (N=7,728)

\begin{tabular}{|c|c|c|c|c|c|c|}
\hline & \multirow{2}{*}{$\begin{array}{c}\text { Total } \\
(\mathrm{N}=7,728)\end{array}$} & \multicolumn{4}{|c|}{ Cholesterol quartiles } & \multirow[b]{2}{*}{$\mathrm{p}$-value for trend } \\
\hline & & $\begin{array}{l}\text { Quartile } 1 \\
(n=1,912)\end{array}$ & $\begin{array}{l}\text { Quartile } 2 \\
(n=1,950)\end{array}$ & $\begin{array}{l}\text { Quartile } 3 \\
(n=1,934)\end{array}$ & $\begin{array}{l}\text { Quartile } 4 \\
(n=1,932)\end{array}$ & \\
\hline \multicolumn{7}{|l|}{ Cholesterol intake (mg/day) } \\
\hline Mean (SD) & $287.1(246.2)$ & $75.9(33.0)$ & $168.1(26.3)$ & $284.2(44.1)$ & $619.1(264.7)$ & \multirow{3}{*}{$<0.001$} \\
\hline Median & 216 & 80.6 & 168 & 279 & 542.8 & \\
\hline IQR & $124.0-373.3$ & $53.0-103.0$ & $145.7-189.8$ & $245.8-319.0$ & $451.0-694.8$ & \\
\hline \multicolumn{7}{|l|}{ Total energy intake (kcal/day) } \\
\hline Mean (SD) & $2,128.8(1,023.2)$ & $1,427.9(642.2)$ & 1,955.2 (703.3) & 2,334.2 (864.3) & $2,791.9(1,239.1)$ & \multirow{3}{*}{$<0.001$} \\
\hline Median & 1,949 & $1,349.5$ & $1,855.6$ & 2,249 & $2,598.7$ & \\
\hline IQR & $1,434.0-2,643.8$ & $994.3-1,755.9$ & $1,480.9-2,309.0$ & $1,736.8-2,855.5$ & $1,905.6-3,407.8$ & \\
\hline \multicolumn{7}{|l|}{ Carbohydrate intake (gm/day) } \\
\hline Mean (SD) & $269.9(136.2)$ & $211.3(106.8)$ & $259.7(114.5)$ & $288.7(131.5)$ & $319.2(161.6)$ & \multirow{3}{*}{$<0.001$} \\
\hline Median & 247 & 195.3 & 242.4 & 268.7 & 293.6 & \\
\hline IQR & $175.0-335.9$ & $136.2-268.0$ & $180.8-316.0$ & $194.2-359.0$ & $209.8-401.4$ & \\
\hline Age (years) & $45.7 \pm 19.6$ & $48.3 \pm 20.7$ & $46.0 \pm 20.1$ & $45.1 \pm 19.2$ & $43.6 \pm 18.2$ & $<0.001$ \\
\hline Male, n (\%) & $3,623(46.9)$ & $628(32.8)$ & $867(44.5)$ & $971(50.2)$ & $1,157(59.9)$ & $<0.001$ \\
\hline \multicolumn{7}{|l|}{ Race, n (\%) } \\
\hline White & $3,724(48.2)$ & $950(49.7)$ & $991(50.8)$ & $952(49.2)$ & $831(43.0)$ & \multirow{5}{*}{0.041} \\
\hline Black & $1,438(18.6)$ & $343(17.9)$ & $336(17.2)$ & $338(17.5)$ & $421(21.8)$ & \\
\hline Mexican & $1,920(24.8)$ & $435(22.8)$ & $469(24.1)$ & $486(25.1)$ & $530(27.4)$ & \\
\hline Other Hispanic & $383(5.0)$ & $102(5.3)$ & $97(5.0)$ & $94(4.9)$ & $90(4.7)$ & \\
\hline Other ethnic & $263(3.4)$ & $82(4.3)$ & $57(2.9)$ & $64(3.3)$ & $60(3.1)$ & \\
\hline $\mathrm{BMl}, \mathrm{kg} / \mathrm{m}^{2}$ & $28.1 \pm 6.3$ & $28.0 \pm 6.3$ & $28.0 \pm 6.4$ & $27.9 \pm 6.0$ & $28.6 \pm 6.7$ & 0.007 \\
\hline Chronic disease history, n (\%) & $2,804(36.3)$ & 759 (39.7) & $720(36.9)$ & $662(34.2)$ & $663(34.3)$ & $<0.001$ \\
\hline $\begin{array}{l}\text { Smoking or passive smoking, } \\
\mathrm{n}(\%)\end{array}$ & $1,604(20.8)$ & $379(19.8)$ & $387(19.8)$ & $394(20.4)$ & $444(23.0)$ & 0.015 \\
\hline \multicolumn{7}{|l|}{ Alcohol intake (gm/day) } \\
\hline 0 & $5,819(75.3)$ & $1,556(81.4)$ & $1,479(75.8)$ & $1,430(73.9)$ & $1,354(70.1)$ & \multirow{3}{*}{$<0.001$} \\
\hline $0-30$ & $1,170(15.1)$ & $237(12.4)$ & $301(15.4)$ & $305(15.8)$ & $327(16.9)$ & \\
\hline$>30$ & $739(9.6)$ & $119(6.2)$ & $170(8.7)$ & $199(10.3)$ & $251(13.0)$ & \\
\hline \multicolumn{7}{|l|}{ Income } \\
\hline Group 1 & $2,664(34.5)$ & $750(39.2)$ & $669(34.3)$ & $616(31.9)$ & $629(32.6)$ & \multirow{3}{*}{$<0.001$} \\
\hline Group 2 & $2,583(33.4)$ & $591(30.9)$ & $679(34.8)$ & $637(32.9)$ & $676(35.0)$ & \\
\hline Group 3 & $2,481(32.1)$ & $571(29.9)$ & $602(30.9)$ & $681(35.2)$ & $627(32.5)$ & \\
\hline \multicolumn{7}{|l|}{ Education } \\
\hline$<$ High school & $2,597(33.6)$ & $692(36.2)$ & $616(31.6)$ & $622(32.2)$ & $667(34.5)$ & \multirow{3}{*}{0.425} \\
\hline High school & $1,826(23.6)$ & $421(22.0)$ & $486(24.9)$ & $483(25.0)$ & $436(22.6)$ & \\
\hline > High school & $3,305(42.8)$ & $799(41.8)$ & $848(43.5)$ & $829(42.9)$ & $829(42.9)$ & \\
\hline \multicolumn{7}{|l|}{ Physical activity (MET-rank) } \\
\hline Very low & $2,027(26.2)$ & $551(28.8)$ & $505(25.9)$ & $463(23.9)$ & $508(26.3)$ & \multirow{4}{*}{0.296} \\
\hline Low & $2,096(27.1)$ & $467(24.4)$ & $550(28.2)$ & $552(28.5)$ & $527(27.3)$ & \\
\hline Moderate & $1,336(17.3)$ & $331(17.3)$ & $344(17.6)$ & $335(17.3)$ & $326(16.9)$ & \\
\hline High & $2,269(29.4)$ & $563(29.4)$ & $551(28.3)$ & $584(30.2)$ & $571(29.6)$ & \\
\hline
\end{tabular}

Cholesterol quartiles: quartile $1<124 \mathrm{mg} /$ day; $124 \leq$ quartile $2<216 \mathrm{mg} /$ day; $216 \leq$ quartile $3<373 \mathrm{mg} /$ day; quartile $4 \geq 373 \mathrm{mg} /$ day; income group1: $\$ 0$ to $\$ 24,999 ;$ group 2: $\$ 25,000$ to $\$ 54,999$; group 3: $\$ 55,000$ and over 
Table 2. Association between cholesterol intake and all-cause mortality

\begin{tabular}{|c|c|c|c|c|}
\hline & \multicolumn{4}{|c|}{ Cholesterol quartiles } \\
\hline & Quartile 1 & Quartile 2 & Quartile 3 & Quartile 4 \\
\hline Outcomes, n (\%) & $171(8.9)$ & $133(6.8)$ & $97(5.0)$ & $118(6.1)$ \\
\hline Follow-up (months) & $68(57-80)$ & $68(57-80)$ & $68(57-80)$ & $69(58-80)$ \\
\hline \multicolumn{5}{|l|}{ Model 1} \\
\hline Crude & $\begin{array}{c}1.81(1.41-2.32) \\
<0.001\end{array}$ & $\begin{array}{c}1.36(1.04-1.76) \\
0.022\end{array}$ & Ref & $\begin{array}{c}1.22(0.93-1.59) \\
0.150\end{array}$ \\
\hline Multivariate* & $\begin{array}{c}1.53(1.16-2.00) \\
0.002\end{array}$ & $\begin{array}{c}1.22(0.94-1.60) \\
0.136\end{array}$ & Ref & $\begin{array}{c}1.39(1.05-1.83) \\
0.020\end{array}$ \\
\hline \multicolumn{5}{|l|}{ Model 2} \\
\hline Crude & Ref & $\begin{array}{c}0.75(0.60-0.94) \\
0.013\end{array}$ & $\begin{array}{c}0.55(0.43-0.71) \\
<0.001\end{array}$ & $\begin{array}{c}0.67(0.53-0.85) \\
<0.001\end{array}$ \\
\hline Multivariate* & Ref & $\begin{array}{c}0.80(0.63-1.02) \\
0.070\end{array}$ & $\begin{array}{c}0.65(0.50-0.86) \\
0.002\end{array}$ & $\begin{array}{c}0.91(0.68-1.21) \\
0.517\end{array}$ \\
\hline
\end{tabular}

Values are percentages (numbers), median (IQR), hazard ratios (95\% Cls), and p-values. *Multivariate models adjusted for total energy, carbohydrate and alcohol intake, age, gender, race, BMI, chronic disease history, smoking status, income, education, and physical activity.

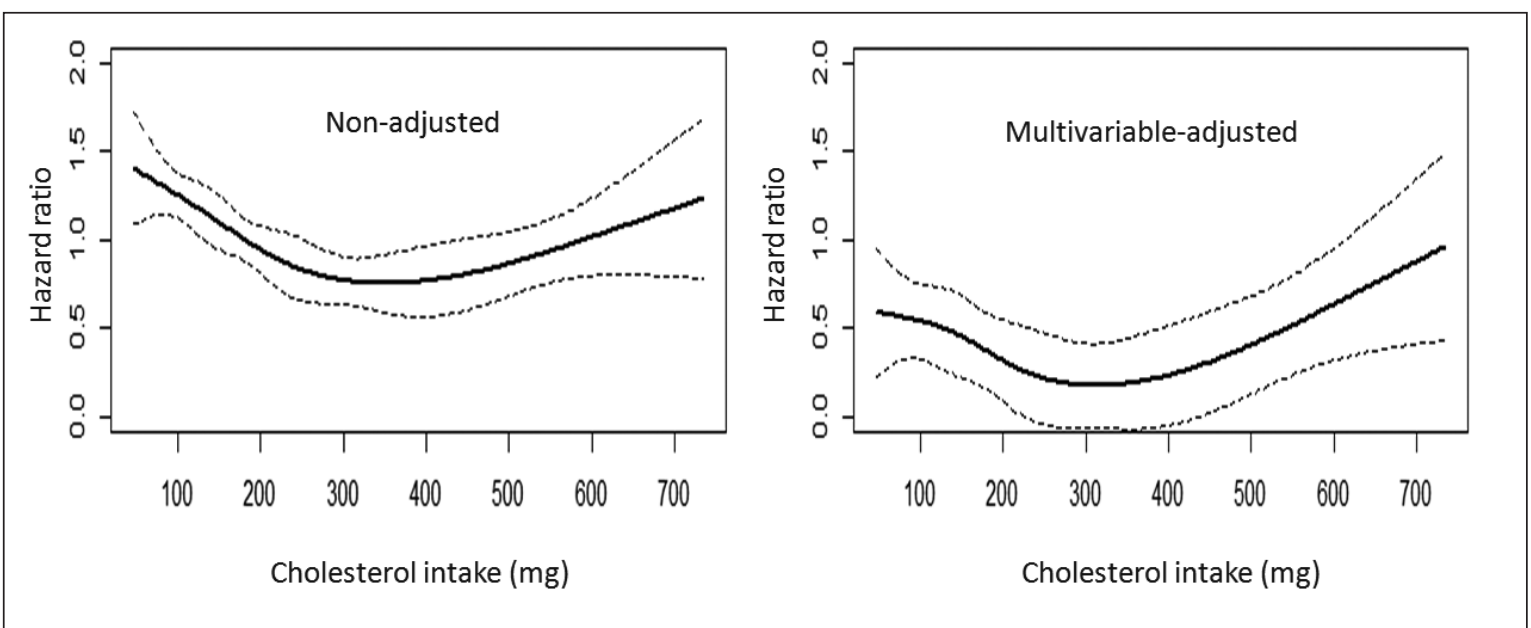

Fig. 1. U-shaped association between cholesterol intake and all-cause mortality.

The top and bottom 5 percentiles of cholesterol intake were excluded.

Asians, thus the generalizability of our findings to Asians requires caution; further studies should analyse the association between cholesterol intake and other major events as well.

\section{CONCLUSION}

In summary, the present study supports an optimal cholesterol intake for lowering the all-cause mortality risk. Healthy dietary cholesterol intake is around $300 \mathrm{mg} /$ day and excessive restrictions of cholesterol intake are harmful to the health.

\section{Acknowledgements}

The present study used an already published NHANES data package, including the 1999-2000, 2001-2002, 2003-2004, and 2005-2006 datasets.

\section{Conflict of Interests}

None declared

\section{REFERENCES}

1. Lewington S, Whitlock G, Clarke R, Sherliker P, Emberson J, Halsey $\mathrm{J}$, et al. Blood cholesterol and vascular mortality by age, sex, and blood pressure: a meta-analysis of individual data from 61 prospective studies with 55,000 vascular deaths. Lancet. 2007;370(9602):1829-39.

2. Weiss A, Beloosesky Y, Schmilovitz-Weiss H, Grossman E, Boaz M. Serum total cholesterol: a mortality predictor in elderly hospitalized patients. Clin Nutr. 2013;32(4):533-7.

3. Stamler J, Daviglus ML, Garside DB, Dyer AR, Greenland P, Neaton JD. Relationship of baseline serum cholesterol levels in 3 large cohorts of younger men to long-term coronary, cardiovascular, and all-cause mortality and to longevity. JAMA. 2000;284(3):311-8.

4. Jakobsen MU, O'reilly EJ, Heitmann BL, Pereira MA, Bälter K, Fraser $\mathrm{GE}$, et al. Major types of dietary fat and risk of coronary heart disease: a pooled analysis of 11 cohort studies. Am J Clin Nutr. 2009;89(5):1425-32.

5. Howard BY, Van Horn L, Hsia J, Manson JE, Stefanick ML, WassertheilSmoller S, et al. Low-fat dietary pattern and risk of cardiovascular disease: the Women's Health Initiative Randomized Controlled Dietary Modification Trial. JAMA. 2006;295(6):655-66.

6. Dehghan M, Mente A, Zhang X, Swaminathan S, Li W, Mohan V, et al. Associations of fats and carbohydrate intake with cardiovascular disease and mortality in 18 countries from five continents (PURE): a prospective cohort study. Lancet. 2017;390(10107):2050-62. 
7. Millen BE, Abrams S, Adams-Campbell L, Anderson CA, Brenna JT, Campbell WW, et al. The 2015 dietary guidelines advisory committee scientific report: development and major conclusions. Adv Nutr. 2016;7(3):438-44.

8. Cogswell ME, Loria CM, Terry AL, Zhao L, Wang CY, Chen TC, et al. Estimated 24-hour urinary sodium and potassium excretion in us adults. JAMA. 2018;319(12):1209-20.

9. Jackson SL, Cogswell ME, Zhao L, Terry AL, Wang CY, Wright J, et al. Association between urinary sodium and potassium excretion and blood pressure among adults in the united states: National health and nutrition examination survey, 2014. Circulation. 2018;137(3):237-46.

10. Patel CJ, Pho N, McDuffie M, Easton-Marks J, Kothari C, Kohane IS, et al. A database of human exposomes and phenomes from the US National Health and Nutrition Examination Survey. Sci Data. 2016;3:160096. doi: 10.1038/sdata.2016.96.

11. Patel CJ, Rehkopf DH, Leppert JT, Bortz WM, Cullen MR, Chertow GM, et al. Systematic evaluation of environmental and behavioural factors associated with all-cause mortality in the united states national health and nutrition examination survey. Int J Epidemiol. 2013;42(6):1795-1810.
12. Nestle M. Perspective: challenges and controversial issues in the dietary guidelines for americans, 1980-2015. Adv Nutr. 2018;9(2):148-50.

13. Lemos BS, Medina-Vera I, Blesso CN, Fernandez ML. Intake of 3 eggs per day when compared to a choline bitartrate supplement, downregulates cholesterol synthesis without changing the LDL/HDL ratio. Nutrients. 2018;10(2):258. doi: 10.3390/nu10020258

14. Ramsden CE, Zamora D, Majchrzak-Hong S, Faurot KR, Broste SK, Frantz RP, et al. Re-evaluation of the traditional diet-heart hypothesis: analysis of recovered data from Minnesota Coronary Experiment (196873). BMJ. 2016;353:i1246. doi: 10.1136/bmj.i1246.

15. Fuller NR, Caterson ID, Sainsbury A, Denyer G, Fong M, Gerofi J, et al. The effect of a high-egg diet on cardiovascular risk factors in people with type 2 diabetes: the diabetes and egg (diabegg) study-a 3-mo randomized controlled trial. Am J Clin Nutr. 2015;101(4):705-13.

Received May 10, 2020 Accepted in revised form May 31, 2021 